\title{
New Descriptions and Analyses of the Third and Fourth Spectra of Zirconium, Zr III and Zr IV
}

\author{
C. C. Kiess
}

\begin{abstract}
Recent observations of the spectra emitted by ionized zirconium atoms have added many new lines to the descriptions of these spectra and have led to revisions and extensions of their term structures. The low, even terms of $\mathrm{Zr}$ III arise in the electron configurations $4 d^{2}, 4 d 5 s$, and $5 s^{2}$. Excitation of these states leads to higher terms of the $4 d 5 p, 5 s 5 p, 4 d$ $5 d, 4 d 6 s, 4 d 4 f, 5 s 5 d, 4 d 6 p, 5 s 6 s, 5 s \quad 4 f$, and $5 s 5 g$ configurations. Most of the singlet and triplet terms from these configurations have now been established. Zeeman patterns from Massachusetts Institute of Technology plates have confirmed and corrected some of the classifications of earlier work. From the series-forming terms of $\mathrm{Zr}$ III the separation of the ground states of $\mathrm{Zr}$ III and $\mathrm{Zr}$ IV is calculated as $198590 \mathrm{~cm}^{-1}$, corresponding to an ionization potential of 24.6 electron volts for the ion $\mathrm{Zr}^{2+}$. In the doublet spectrum of $\mathrm{Zr}$ IV, which arises from the migration of the single electron outside the krypton shell, the terms from the $7 s, 6 p, 6 d, 5 f, 6 f, 5 g$, and $6 g$ electrons have been added to those already known. A mean value of $276970 \mathrm{~cm}^{-1}$ for the separation of the ground states of $\mathrm{Zr}$ IV and $\mathrm{Zr} \mathrm{v}$ has been derived from three accordant values calculated from series of ${ }^{2} \mathrm{~S},{ }^{2} \mathrm{~F}^{\circ}$, and ${ }^{2} \mathrm{G}$ terms. This corresponds to an ionization potential of 34.33 electron volts for $\mathrm{Zr}^{3+}$.
\end{abstract}

\section{Introduction}

The new descriptions of the third and fourth spectra of zirconium, presented in this paper, have led to a reexamination of the analyses of their term structures published 25 years ago by Kiess and Lang [1]. ${ }^{1} \quad$ Recent observations have more than doubled the number of lines known for these spectra at that time, and studies of the Zeeman effect have given unmistakable information about the classification of some of the lines. Preliminary reports [2] on the application of the new results to optical and astrophysical problems have already been made, and a paper has been published [3] with the new additions to and interpretations of the second spectrum, $\mathrm{Zr}$ II.

\section{Experimental Procedure}

Spectra of neutral and ionized zirconium atoms were obtained with a concave-grating spectrograph at the National Bureau of Standards, down to $1920 \mathrm{~A}$ in the ultraviolet. The grating, of $21-\mathrm{ft}$ radius of curvature, was ruled by $R$. W. Wood [4] with 30,000 lines per inch. It is set up in a Wadsworth mounting and gives uniformly bright spectra throughout the accessible first and second orders, with a dispersion of $2 \mathrm{~A} / \mathrm{mm}$ in the first order. The spectra were emitted by arcs and condensed-spark discharges in air between electrodes of the same zirconium metal that was used in the earlier work. Although of high purity this metal was found to contain very small amounts of silicon, titanium, iron, and hafnium. The arcs were operated at about 5 amp from 220 -volt d-c mains. The sparks were excited by a battery of condensers, of $0.006-\mu \mathrm{f}$ capacitance, charged from the high-voltage side of a transformer that stepped-up 110 volts a-c to 30,000 volts. The plates used to record the spectra were

\footnotetext{
1 Figures in brackets indicate the literature references at the end of this paper.
}

coated with EK 33 and SWR emulsions. Each exposure to a zirconium spectrum was juxtaposed to an exposure of the iron are or to the spark between copper-silver electrodes to secure the necessary standards for the wavelength calculations.

For the extreme ultraviolet region of the spectra two sets of spectrograms were available for measurement, one obtained with the Carnegie vacuum spectrograph by J. C. Boyce while he was at the Massachusetts Institute of Technology, the other by A. G. Shenstone with the Princeton vacuum spectrograph. Both of these instruments have the same optical dimensions and produce spectra with dispersions of $4.25 \mathrm{~A} / \mathrm{mm}$ in the first order from concave gratings ruled with 30,000 lines per inch. The results from these new measurements have confirmed and supplemented the descriptions of the ultraviolet spectra of $\mathrm{Zr}$ supplied by R. J. Lang for the Bureau's work of 25 years ago.

The MIT spectrograms were obtained from exposures to an arc operated at $8 \mathrm{amp}$ between $\mathrm{Zr}-\mathrm{Ag}$ electrodes in an atmosphere of commercial $\mathrm{N}_{2}$, and cover the region from $2500 \mathrm{~A}$ down to $1100 \mathrm{~A}$. The recorded spectra are mixtures of lines of zirconium, silver, nitrogen, carbon, and hafnium. The Princeton plates cover the region from $2200 \mathrm{~A}$ down to 513 A with exposures to a condensed-spark discharge between $\mathrm{Zr}$ electrodes in vacuum. On both sets of spectrograms appear lines of $\mathrm{Zr}$ I, $\mathrm{Zr}$ II, Zr III, and $\mathrm{Zr}$ IV. On the MIT plates the lines all exhibit uniform distributions of illumination along the slit of the spectrograph and differ only in their intensities and qualities of sharpness and diffuseness. On the Princeton plates, however, the lines exhibit distinctive differences of illumination along the slit of the spectrograph that permit them to be grouped according to the degree of ionization of their emitters: those of uniform intensity along the slit belong mostly to $\mathrm{Zr}$ i ; lines of $\mathrm{Zr}$ is generally show illumination along the full length of the slit but are stronger 
near the electrodes than in the zone midway between them; lines strong at the electrodes and directed like spear-points toward the center of the slit are characteristic of Zr III; while lines strong at the electrodes but tapering outward along the slit belong to $\mathrm{Zr}$ IV, and perhaps to $\mathrm{Zr}$ v. There are about 50 lines of this latter kind in the region between 900 and $600 \mathrm{~A}$ for which there is no place in $\mathrm{Zr}$ IV. It is very likely that they represent the excitation of the Kr-shell of the zirconium atom.

Preliminary values of the wavelengths derived from measurement of the MIT and Princeton plates were based on standards selected from lines of $\mathrm{N}, \mathrm{O}, \mathrm{C}$, and $\mathrm{H}$ that appear as impurities in the spectra. These wavelengths were of sufficient accuracy to allow assignment of many of the lines to their multiplet structures. With more accurate values for energy levels calculated from lines longer than $2000 \mathrm{~A}$, which were measured against better standards than those in the vacuum region, corrections were derived for the preliminary wavelengths longer than $1200 \mathrm{~A}$. Nearly all the strong lines with wavelengths shorter than $1200 \mathrm{~A}$ appeared in the second and third orders, overlapping the first order from 2400 to $1000 \mathrm{~A}$. Their apparent wavelengths, after being corrected as just described, were then divided by the appropriate factor to give the adopted values in the first-order spectrum.

There were also available for measurement plates of the Zeeman effect obtained under the direction of G. R. Harrison at MIT. Although most of the magnetic patterns appearing on these plates are of $\mathrm{Zr}$ I and $\mathrm{Zr}$ II lines, yet a few of them belong to lines of $\mathrm{Zr}$ III. These serve to verify and correct some of the earlier classifications. The results of the measurements are given below.

\section{Results}

\subsection{Spectrum of Trebly Ionized Zirconium, Zr IV}

In its unexcited state the neutral zirconium atom has four valence electrons in the configuration $4 d^{2} 5 s^{2}$ outside the krypton shell. The loss of three of these electrons through successive ionizations leaves a single $4 d$-electron to determine the term-structure of $\mathrm{Zr}$ IV. The early work on the analysis of $\mathrm{Zr}$ iv is summarized in the paper by Kiess and Lang cited above. The terms listed therein account for 12 lines of the spectrum if the term designated there as $7 s$, and now known to be incorrect, is excluded. In the present analysis the 39 lines given in table 1 are classified as combinations among the 21 terms recorded in table 2 , and include all lines classified in the earlier investigations.

The terms of $\mathrm{Zr} \mathrm{IV}$, in table 2, are listed in the order of increasing energy indicated by the relative term-values in the second column and shown graphically in figure 1 . In the fourth and fifth columns are given the absolute values of the terms, and the Rydberg denominators that were used in the simple Ritz formula $16 R /\left[m+\alpha+\left(\beta / m^{2}\right)\right]^{2}$ to evaluate the running terms and the limits of the several series. Inspection of the table shows that three members have been found for the ${ }^{2} \mathrm{~S},{ }^{2} \mathrm{D}$, and ${ }^{2} \mathrm{~F}^{\circ}$ series, and two members for the ${ }^{2} \mathrm{P}^{\circ}$ and ${ }^{2} \mathrm{G}$ series. The terms of the three-member series are accurately represented by the above formula with the appropriate values of $\alpha$ and $\beta$ introduced into it. For the two-member series the terms were interpolated from the Princeton table for Rydberg's formula [5]. Table 3 shows that the values for the ground state $4 p{ }^{2} \mathrm{D}_{1 \frac{1}{2}}$ of $\mathrm{Zr}$ Iv given by the ${ }^{2} \mathrm{~S},{ }^{2} \mathrm{~F}^{\circ}$, and the nearly hydrogenic ${ }^{2} \mathrm{G}$ series are in close agreement, whereas the ${ }^{2} \mathrm{P}^{\circ}$ and ${ }^{2} \mathrm{D}$ series give much larger values. It is known from other spectra that series terms from

TaBle 1. Classified lines of Zr IV

Wavelengths longer than $2000 \mathrm{~A}$, air values; vacuum values shorter than $2000 \mathrm{~A}$

\begin{tabular}{|c|c|c|c|}
\hline $\begin{array}{l}\text { Wave- } \\
\text { lengths }\end{array}$ & $\begin{array}{l}\text { Inten- } \\
\text { sities }\end{array}$ & $\begin{array}{l}\text { Wave } \\
\text { numbers }\end{array}$ & Term combinations \\
\hline$A$ & & $K\left(\mathrm{~cm}^{-1}\right)$ & \\
\hline 2849. 19 & 10 & 35087. 41 & $6 p^{2} \mathrm{P}_{11 / 2}^{\circ}-6 d^{2} \mathrm{D}_{11 / 2}$ \\
\hline 2832. 82 & 15 & 35290.15 & $6 p{ }^{2} \mathrm{P}_{11 / 2}^{1 / 2}-6 d{ }^{2} \mathrm{D}_{21 / 2}$ \\
\hline 2770.44 & 5 & 36084. 72 & $6 p^{2} \mathrm{P}_{01 / 2}^{1 / 2}-6 d^{2} \mathrm{D}_{11 / 2}$ \\
\hline 2286.68 & 200 & 43718. 01 & $5 s^{2} \mathrm{~S}_{01 / 2}-5 p{ }^{2} \mathrm{P}_{01 / 2}^{\circ}$ \\
\hline 2163. 63 & 500 & 46204.06 & $5 s^{2} \mathrm{~S}_{01 / 2}-5 p{ }^{2} \mathrm{P}_{11 / 2}^{0 / 1 / 2}$ \\
\hline 2125. 29 & 25 & 47037. 48 & $4 f^{2} \mathrm{~F}_{21 / 2}^{\circ}-6 d{ }^{2} \mathrm{D}_{21 / 2}$ \\
\hline 2092. 40 & 150 & 47776.76 & $4 f^{2} \mathrm{~F}_{31 / 2}^{\circ}-5 g{ }^{2} \mathrm{G}_{41 / 2}$ \\
\hline 2091. 49 & 125 & 47797.54 & $4 f^{2} \mathrm{~F}_{21 / 2}^{\circ / 2}-5 g{ }^{2} \mathrm{G}_{31 / 2}$ \\
\hline 1848. 06 & 10 & 54110.8 & $5 d^{2} \mathrm{D}_{21 / 2}-5 f{ }^{2} \mathrm{~F}_{21 / 2}^{\circ}$ \\
\hline 1846. 42 & 100 & 54158. 9 & $5 d^{2} \mathrm{D}_{21 / 2}-5 f{ }^{2} \mathrm{~F}_{31 / 2}^{\circ}$ \\
\hline 1836. 14 & 100 & 54462.1 & $5 d^{2} \mathrm{D}_{11 / 2}-5 f{ }^{2} \mathrm{~F}_{21 / 2}^{\circ}$ \\
\hline 1607.99 & 100 & 62189.4 & $5 p^{2} \mathrm{P}_{11 / 2}^{\circ}-5 d^{2} \mathrm{D}_{11 / 2}$ \\
\hline 1598.98 & 200 & 62539.9 & $5 p^{2} \mathrm{P}_{11 / 2}^{11 / 2}-5 d^{2} \mathrm{D}_{21 / 2}$ \\
\hline 1546. 19 & 150 & 64675.1 & $5 p^{2} \mathrm{P}_{01 / 2}^{1 / 2}-5 d^{2} \mathrm{D}_{11 / 2}$ \\
\hline 1469.50 & 100 & 68050.4 & $5 p{ }^{2} \mathbf{P}_{11 / 2}^{\circ}-6 s{ }^{2} \mathrm{~S}_{01 / 2}$ \\
\hline 1441. 11 & 20 & 69391.0 & $4 f^{2} \mathrm{~F}_{31 / 2}^{18}-6 g^{2} \mathrm{G}_{41 / 2}^{\circ}$ \\
\hline 1440.68 & 18 & 69411. 7 & $4 f^{2} \mathrm{~F}_{21 / 2}^{\circ}-6 g^{2} \mathrm{G}_{31 / 2}$ \\
\hline 1417.73 & 75 & 70535.3 & $5 p^{2} \mathrm{P}_{01 / 2}^{0}-6 s{ }^{2} \mathrm{~S}_{01 / 2}$ \\
\hline 1291. 77 & 1 & 77413. 1 & $5 d{ }^{2} \mathrm{D}_{21 / 2}-6 f{ }^{2} \mathrm{~F}_{21 / 2}^{\circ}$ \\
\hline 1290. 60 & 20 & 77483.3 & $5 d^{2} \mathrm{D}_{21 / 2}-6 f{ }^{2} \mathrm{~F}_{31 / 2}^{0}$ \\
\hline 1285. 94 & 15 & 77764.1 & $5 d^{2} \mathrm{D}_{11 / 2}-6 f^{2} \mathrm{~F}_{21 / 2}^{0}$ \\
\hline 1219. 85 & 150 & 81977. 3 & $4 d^{2} \mathrm{D}_{11 / 2}-5 p{ }^{2} \mathrm{P}_{01 / 2}^{\circ}$ \\
\hline 1201. 77 & 250 & 83210.6 & $4 d^{2} \mathrm{D}_{21 / 2}-5 p{ }^{2} \mathrm{P}_{11 / 2}^{\circ}$ \\
\hline 1183. 96 & 100 & 84462.3 & $4 d^{2} \mathrm{D}_{11 / 2}-5 p^{2} \mathrm{P}_{11 / 2}$ \\
\hline 864.61 & 8 & 115659 & $5 p^{2} \mathrm{P}_{1 \zeta a}^{\circ}-7 s{ }^{2} \mathrm{~S}_{01 / 2}$ \\
\hline 846. 42 & 4 & 118145 & $5 p{ }^{2} \mathbf{P}_{01 / 2}^{0}-7 s{ }^{2} \mathbf{S}_{01 / 2}$ \\
\hline 823.50 & 20 & 121433 & $5 p{ }^{2} \mathrm{P}_{11 / 2}^{\circ}-6 d^{2} \mathrm{D}_{11 / 2}$ \\
\hline 822.09 & 20 & 121647 & $5 p{ }^{2} \mathrm{P}_{11 / 2}^{11 / 2}-6 d^{2} \mathrm{D}_{21 / 2}$ \\
\hline 806.98 & 20 & 123919 & $5 p{ }^{2} \mathrm{P}_{01 / 2}^{1}-6 d^{2} \mathrm{D}_{11 / 2}$ \\
\hline 760.18 & 8 & 131548 & $5 s^{2} \mathrm{~S}_{01 / 2}-6 p{ }^{2} \mathrm{P}_{01 / 2}^{\circ}$ \\
\hline 754.42 & 10 & 132552 & $5 s^{2} \mathrm{~S}_{31 / 2}-6 p^{2} \mathrm{P}_{11 / 2}^{0 / 2}$ \\
\hline 633.66 & 20 & 157813 & $4 d{ }^{2} \mathrm{D}_{21 / 2}-4 f{ }^{2} \mathrm{~F}_{21 / 2}^{\circ}$ \\
\hline 633.58 & 100 & 157833 & $4 d^{2} \mathrm{D}_{21 / 2}-4 f^{2} \mathrm{~F}_{31 / 2}^{2012}$ \\
\hline 628.68 & 100 & 159063 & $4 d^{2} \mathrm{D}_{11 / 2}-4 f{ }^{2} \mathrm{~F}_{21 / 2}^{\circ / 2}$ \\
\hline 589.75 & & & \\
\hline 588. 89 & 25 & 169563 & $4 d^{2} \mathrm{D}_{21 / 2}-6 p{ }^{2} \mathrm{P}_{11 / 2}^{\circ}$ \\
\hline 585. 43 & $\begin{array}{r}20 \\
5\end{array}$ & $\begin{array}{l}169811 \\
170815\end{array}$ & $\begin{array}{l}4 d^{2} \mathrm{D}_{11 / 2}-6 p{ }^{2} \mathrm{P}_{01 / 2} \\
4 d^{2} \mathrm{D}_{11 / 2}-6 p{ }^{2} \mathrm{P}_{11 / 2}^{0}\end{array}$ \\
\hline 500.22 & & & \\
\hline 497. 12 & $\begin{array}{l}2 \\
1\end{array}$ & $\begin{array}{l}199910 \\
201117\end{array}$ & $\begin{array}{l}4 d^{2} \mathrm{D}_{21 / 2}-5 f{ }^{2} \mathrm{~F}_{31 / 2}^{\circ} \\
4 d^{2}{ }^{2} \mathrm{D}_{11 / 2}-5 f \\
{ }^{2} \mathrm{~F}_{21 / 2}^{\circ}\end{array}$ \\
\hline
\end{tabular}


the $p$ and $d$ electrons usually lead to limits that are too large; but the difference of $18000 \mathrm{~cm}^{-1}$ between the values for the limit from the ${ }^{2} \mathrm{D}$ and ${ }^{2} \mathrm{~S}$ series of $\mathrm{Zr}$ Iv appears to be excessively large. The value adopted, therefore, for the ground state of $\mathrm{Zr}$ IV is the mean of the three accordant determinations, namely, $276970 \mathrm{~cm}^{-1}$. It yields an ionization potential of $34.33 \mathrm{ev}$ for the $\mathrm{Zr}^{3+}$ ion.

TABLE 2. Terms of $\mathrm{Zr}$ IV

\begin{tabular}{|c|c|c|c|c|}
\hline $\begin{array}{l}\text { Term desig- } \\
\text { nations }\end{array}$ & $\begin{array}{l}\text { Relative } \\
\text { term } \\
\text { values }\end{array}$ & $\begin{array}{l}\text { Term } \\
\text { separa- } \\
\text { tions }\end{array}$ & $\begin{array}{l}\text { Absolute } \\
\text { term } \\
\text { values }\end{array}$ & $\begin{array}{c}\text { Rydberg } \\
\text { denomi- } \\
\text { nators }\end{array}$ \\
\hline $4 d\left\{\begin{array}{l}2 \mathrm{D}_{11 / 2} \\
2 \mathrm{D}_{21 / 2}\end{array}\right.$ & $\begin{array}{r}0 \\
1250\end{array}$ & 1250 & $\begin{array}{l}276970 \\
275720\end{array}$ & $\begin{array}{l}\text { 2. } 5171 \\
\text { 2. } 5228\end{array}$ \\
\hline $5 s{ }^{2} \mathrm{~S}_{01 / 2}$ & 38258 & & 238712 & 2. 7113 \\
\hline $5 p\left\{\begin{array}{l}2 \mathrm{P}_{01 / 2}^{\circ} \\
{ }^{2} \mathrm{P}_{11 / 2}^{\circ}\end{array}\right.$ & $\begin{array}{l}81977 \\
84461\end{array}$ & 2484 & $\begin{array}{l}194993 \\
192509\end{array}$ & $\begin{array}{l}\text { 2. } 9999 \\
\text { 3. } 0192\end{array}$ \\
\hline $5 d\left\{\begin{array}{l}2 \mathrm{D}_{11 / 2} \\
2 \mathrm{D}_{21 / 2}\end{array}\right.$ & $\begin{array}{l}146651 \\
147001\end{array}$ & 350 & $\begin{array}{l}130319 \\
129969\end{array}$ & $\begin{array}{l}\text { 3. } 6696 \\
\text { 3. } 6745\end{array}$ \\
\hline $6 s^{2} \mathbf{S}_{01 / 2}$ & 152511 & & 124459 & 3. 7550 \\
\hline $4 f\left\{\begin{array}{l}2 \mathrm{~F}_{21 / 2}^{\circ} \\
{ }^{2} \mathrm{~F}_{31 / 2}^{\circ}\end{array}\right.$ & $\begin{array}{l}159063 \\
159083\end{array}$ & 20 & $\begin{array}{l}117907 \\
117887\end{array}$ & $\begin{array}{l}\text { 3. } 8579 \\
\text { 3. } 8582\end{array}$ \\
\hline $6 p\left\{\begin{array}{l}2 \mathrm{P}_{01 / 2}^{\circ} \\
{ }^{2} \mathrm{P}_{11 / 2}^{\circ}\end{array}\right.$ & $\begin{array}{l}169805 \\
170810\end{array}$ & 1005 & $\begin{array}{l}107165 \\
106160\end{array}$ & $\begin{array}{l}\text { 4. } 0466 \\
\text { 4. } 0657\end{array}$ \\
\hline $7 s^{2} \mathbf{S}_{03 / 2}$ & 200121 & & 76849 & 4. 7786 \\
\hline $5 f\left\{\begin{array}{l}2 \mathrm{~F}_{21 / 2}^{\circ} \\
2 \mathrm{~F}_{31 / 2}^{\circ}\end{array}\right.$ & $\begin{array}{l}201112 \\
201160\end{array}$ & 48 & $\begin{array}{l}75858 \\
75810\end{array}$ & $\begin{array}{l}\text { 4. } 8097 \\
\text { 4. } 8112\end{array}$ \\
\hline $6 d\left\{\begin{array}{l}2 \mathrm{D}_{11 / 2} \\
2 \mathrm{D}_{21 / 2}\end{array}\right.$ & $\begin{array}{l}205896 \\
206102\end{array}$ & 206 & $\begin{array}{l}71074 \\
70868\end{array}$ & $\begin{array}{l}\text { 4. } 9689 \\
\text { 4. } 9762\end{array}$ \\
\hline $5 g^{2} \mathrm{G}_{41 / 2}, 31 / 2$ & 206860 & & 70110 & 5. 0030 \\
\hline $6 f\left\{\begin{array}{l}2 \mathrm{~F}_{21 / 2}^{\circ} \\
{ }^{2} \mathrm{~F}_{31 / 2}^{\circ}\end{array}\right.$ & $\begin{array}{l}224415 \\
224484\end{array}$ & 69 & $\begin{array}{l}52555 \\
52486\end{array}$ & $\begin{array}{l}\text { 5. } 7785 \\
\text { 5. } 7823\end{array}$ \\
\hline $6 g^{2} \mathrm{G}_{41 / 2}, 31 / 2$ & 228474 & & 48496 & 6. 0154 \\
\hline
\end{tabular}

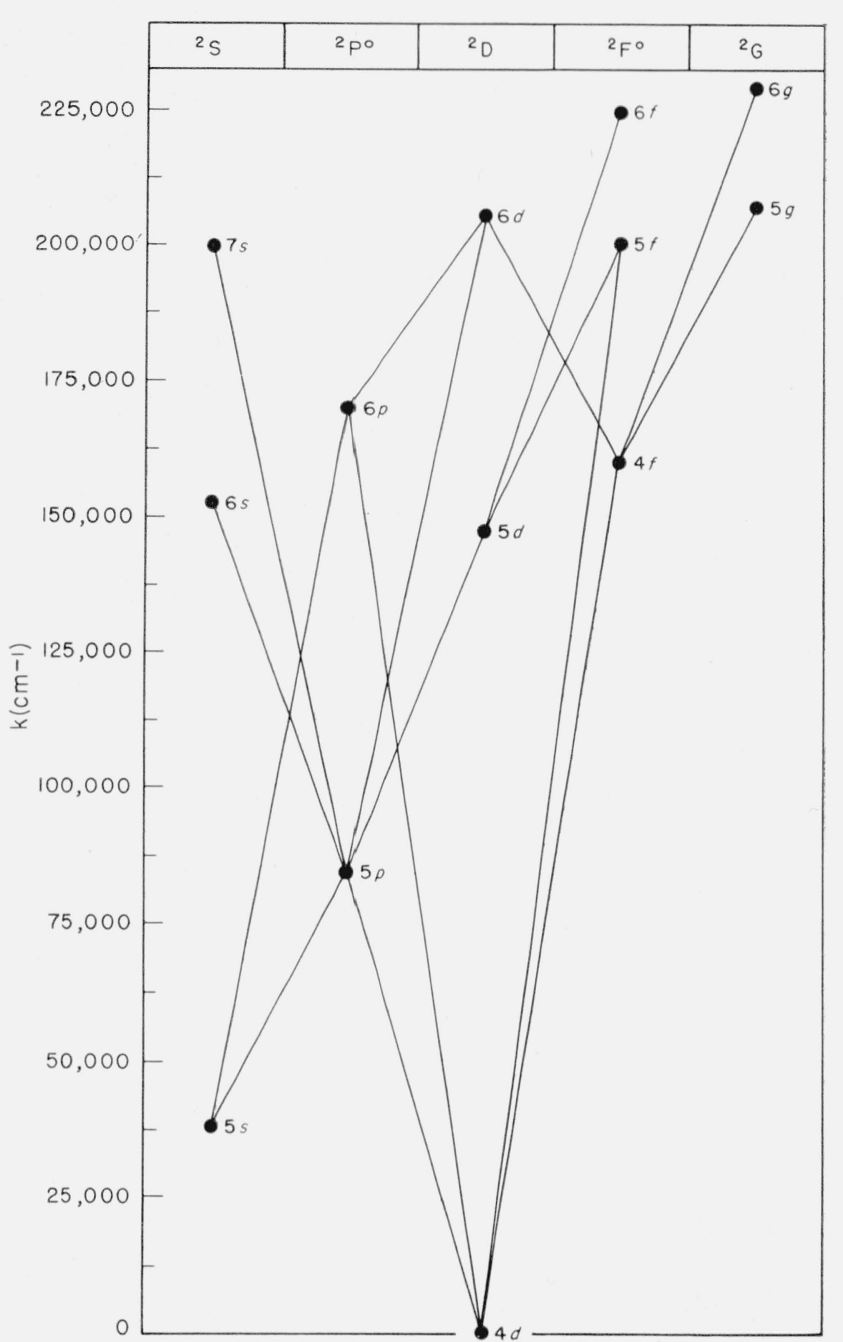

FIgure 1. Grotrian diagram of the terms and combinations of Zr IV.

Table 3. Series of $\mathrm{Zr}$ IV

\begin{tabular}{|c|c|c|c|c|c|}
\hline \multirow{2}{*}{ Evaluation of limit } & \multicolumn{5}{|c|}{ Series } \\
\hline & ${ }^{2} \mathrm{~S}$ & ${ }^{2} \mathrm{P}^{\circ}$ & ${ }^{2} \mathrm{D}$ & ${ }^{2} \mathrm{~F}^{\circ}$ & ${ }^{2} \mathrm{G}$ \\
\hline Limit term $\ldots \ldots . .$. & $5 p^{2} \mathrm{P}^{\circ}$ & $4 d^{2} \mathrm{D}$ & $5 p^{2} \mathrm{P}^{\circ}$ & $4 d^{2} \mathrm{D}$ & $4 f^{2} \mathrm{~F}^{\circ}$ \\
\hline Value of limit term $\ldots \ldots$ & 194735 & 281340 & 212850 & 276734 & 118396 \\
\hline Separation of limit term from ground state $\ldots$ & 81977 & 0 & 81977 & 0 & 159063 \\
\hline Absolute value of $4 p^{2} \mathrm{D}_{11 / 2-\ldots-}$ & 276712 & 281340 & 294827 & 276734 & 277459 \\
\hline - n- & -2.1364 & & -1.5558 & -0.2577 & \\
\hline$\beta_{--}$ & -3.7706 & & -0.0730 & +1.9133 & \\
\hline $\begin{array}{l}\text { Adopted mean separation of } \\
\text { Ionization potential of } \mathrm{Zr}^{3+}=\end{array}$ & $\begin{array}{l}\text { ground } \\
.33 \mathrm{ev} \text {. }\end{array}$ & of $\mathrm{Zr} \mathrm{v}$ & $V=2769$ & $n^{-1}$ & \\
\hline
\end{tabular}


TABLE 4. Theoretical terms of $\mathrm{Zr}$ III

\begin{tabular}{|c|c|c|c|c|c|c|c|c|c|c|c|c|}
\hline $\begin{array}{c}\text { Electron } \\
\text { configurations }\end{array}$ & \multicolumn{11}{|c|}{ Terms } & Limit \\
\hline $\begin{array}{ll}4 d & 4 d \\
4 d & 5 s \\
5 s & 5 s \\
4 d & 5 p \\
5 s & 5 p \\
4 d & 5 d \\
4 d & 6 s \\
4 d & 4 f \\
5 p & 5 p \\
5 s & 5 d \\
5 s & 6 s \\
4 d & 6 p \\
5 s & 4 f \\
5 s & 5 g\end{array}$ & $\begin{array}{l}{ }^{1} \mathrm{~S} \\
{ }^{1} \mathrm{~S} \\
{ }^{1} \mathrm{~S}\end{array}$ & $\begin{array}{l}{ }^{1} \mathrm{P}^{\circ} \\
{ }^{1} \mathrm{P}^{\circ} \\
{ }^{1} \mathrm{P}^{1} \mathrm{P}^{\circ} \\
{ }^{1} \mathrm{P}^{\circ}\end{array}$ & $\begin{array}{l}{ }^{1} \mathrm{D} \\
{ }^{1} \mathrm{D} \\
{ }^{1} \mathrm{D}^{\circ} \\
{ }^{1} \mathrm{D} \\
{ }^{1} \mathrm{D} \\
{ }^{1} \mathrm{D}^{\circ} \\
{ }^{1} \mathrm{D}^{1} \mathrm{D} \\
{ }^{1} \mathrm{D}\end{array}$ & $\begin{array}{l}{ }^{1} \mathrm{~F}^{\circ} \\
{ }^{1} \mathrm{~F} \\
{ }^{1} \mathrm{~F}^{\circ} \\
{ }^{1} \mathrm{~F}^{\circ}\end{array}$ & $\begin{array}{l}{ }^{1} \mathrm{G} \\
{ }^{1} \mathrm{G}^{\circ} \\
{ }^{1} \mathrm{G}\end{array}$ & ${ }^{1} \mathrm{H}^{\circ}$ & ${ }^{3} \mathrm{~S}$ & $\begin{array}{l}{ }^{3} \mathrm{P} \\
{ }^{3} \mathrm{P}^{\circ} \\
{ }^{3} \mathrm{P}^{\circ} \\
{ }^{3} \mathrm{P} \\
{ }^{3} \mathrm{P}^{\circ} \\
{ }^{3} \mathrm{P}^{\circ} \\
{ }^{3} \mathrm{P}^{\circ}\end{array}$ & $\begin{array}{l}{ }^{3} \mathrm{D} \\
{ }^{3} \mathrm{D}^{\circ} \\
{ }^{3} \mathrm{D} \\
{ }^{3} \mathrm{D}^{\circ} \\
{ }^{3} \mathrm{D}^{\circ} \\
{ }^{3} \mathrm{D} \\
{ }^{3} \mathrm{D}^{\circ}\end{array}$ & $\begin{array}{l}{ }^{3} \mathrm{~F} \\
{ }^{3} \mathrm{~F}^{\circ} \\
{ }^{3} \mathrm{~F} \\
{ }^{3} \mathrm{~F}^{\circ} \\
{ }^{3} \mathrm{~F}^{\circ} \\
{ }^{3} \mathrm{~F}^{\circ}\end{array}$ & $\begin{array}{l}{ }^{3} \mathrm{G} \\
{ }^{3} \mathrm{G}^{\circ} \quad{ }^{3} \mathrm{H}^{\circ} \\
{ }^{3} \mathrm{G}\end{array}$ & $\begin{array}{l}{ }^{2} \mathrm{D} \\
{ }^{2} \mathrm{D} \\
{ }^{2} \mathrm{~S} \\
{ }^{2} \mathrm{D} \\
{ }^{2} \mathrm{~S} \\
{ }^{2} \mathrm{D} \\
{ }^{2} \mathrm{D} \\
{ }^{2} \mathrm{D} \\
{ }^{2} \mathrm{P} \\
{ }^{2} \mathrm{~S} \\
{ }^{2} \mathrm{~S} \\
{ }^{2} \mathrm{D} \\
{ }^{2} \mathrm{~S} \\
{ }^{2} \mathrm{~S}\end{array}$ \\
\hline
\end{tabular}

\subsection{Spectrum of Doubly Ionized Zirconium, $\mathrm{Zr}$}

The term structure of the third spectrum of zirconium presented in the earlier work pertains only to that portion that is derived by addition of $n s$, $n p, n d$, and $n f$ electrons to the ground term $4 d^{2} \mathrm{D}$ of $\mathrm{Zr}$ IV. However, other terms are to be expected from the addition of similar and also $n g$ electrons to the excited states $5 s^{2} \mathrm{~S}$ and $5 p^{2} \mathrm{P}^{\circ}$ of $\mathrm{Zr}$ Iv. All of such theoretical terms that have been found in the present investigation are given in table 4, along with the previously known terms. The level-values of

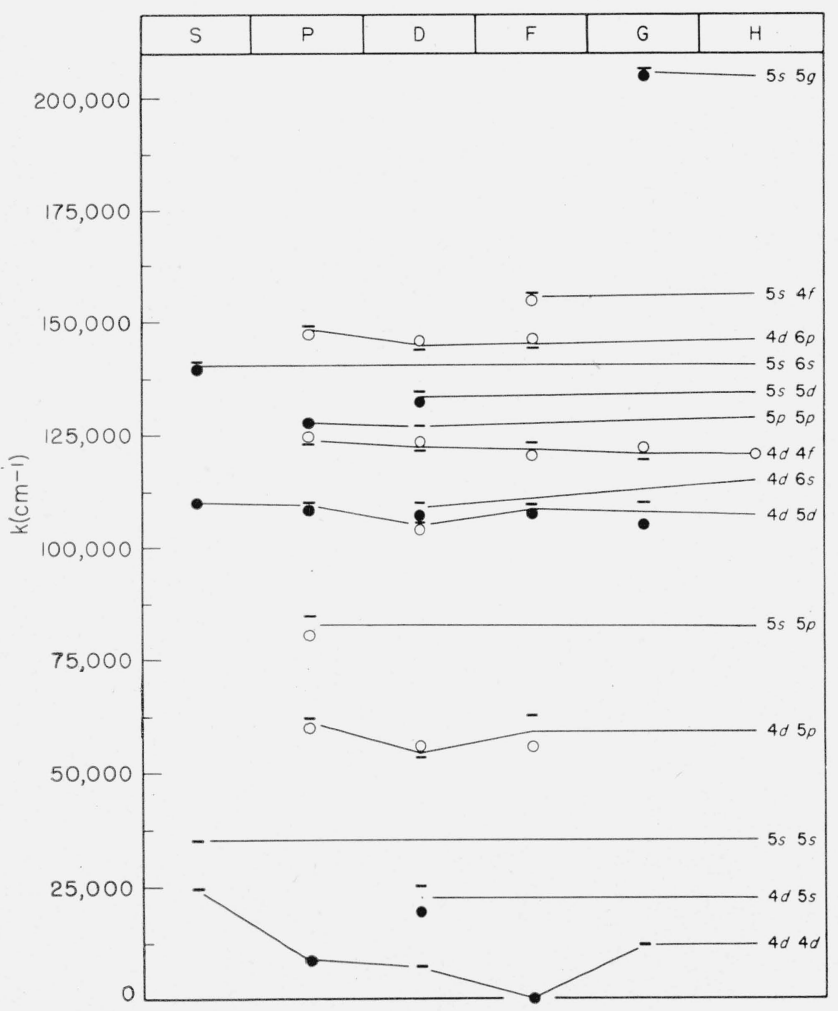

Figure 2. Term diagram of $\mathrm{Zr}$ III.

- Even triplet terms; $\bigcirc$, odd triplet terms; -, singlet terms. these terms are given in table 5, and all the lines classified as allowed transitions among the levels are listed in table 6 . The relative positions of the Zr nI terms are shown in figure 2. For some of the classified lines of $\mathrm{Zr} ı I I$, Zeeman patterns were found on the MIT spectrograms of zirconium. Although few in number these patterns verify some and correct other classifications of lines in the earlier work. They are given in table 7 .

Among the terms in table 5 are several pairs that are parts of Rydberg series. No sequence of three or more members of a series has been found in $\mathrm{Zr}$ 11I. Nine such pairs of terms and their absolute values, as interpolated from the Princeton table, are recorded in table 8. By adding to each its distance above the ground state $4 d{ }^{3} \mathrm{~F}_{2}$ of $\mathrm{Zr}$ III, a value for the gound state may be derived. Inspection of the table shows that also with $\mathrm{Zr}$ III the terms from configurations containing $p$-electrons give higher values for the ground state than do the others. The values for $4 d^{3} \mathbf{F}_{2}$ from configurations with running $n s$ and $n d$ electrons are in good agreement, and have been averaged to give a mean value of $198590 \mathrm{~cm}^{-1}$ for the separation of the ground state of Zr III from that of Zriv. This corresponds to an ionization potential of $24.6 \mathrm{ev}$.

TaBLE 5. Terms of Zr III

\begin{tabular}{|c|c|c|c|}
\hline $\begin{array}{l}\text { Electron con- } \\
\text { figuration }\end{array}$ & Term & $\begin{array}{c}\text { Relative term } \\
\text { value }\end{array}$ & $\begin{array}{l}\text { Term sepa- } \\
\text { ration }\end{array}$ \\
\hline $4 d\left({ }^{2} \mathrm{D}\right) 4 d$ & $\begin{array}{l}{ }^{3} \mathrm{~F}_{2} \\
{ }^{3} \mathrm{~F}_{3} \\
{ }^{3} \mathrm{~F}_{4} \\
{ }^{1} \mathrm{D} \\
{ }^{3} \mathrm{P}_{0} \\
{ }^{3} \mathrm{P}_{1} \\
{ }^{3} \mathrm{P}_{2} \\
{ }^{1} \mathrm{G}_{4} \\
{ }^{1} \mathrm{~S}_{0}\end{array}$ & $\begin{array}{r}0.0 \\
680.5 \\
1485.7 \\
5740.9 \\
8061.50 \\
8324.92 \\
8837.45 \\
11047.90 \\
23976.48\end{array}$ & $\begin{array}{l}\text { 263. } 42 \\
512.53\end{array}$ \\
\hline
\end{tabular}


TABle 5. Terms of $\mathrm{Zr}$ III-Continued

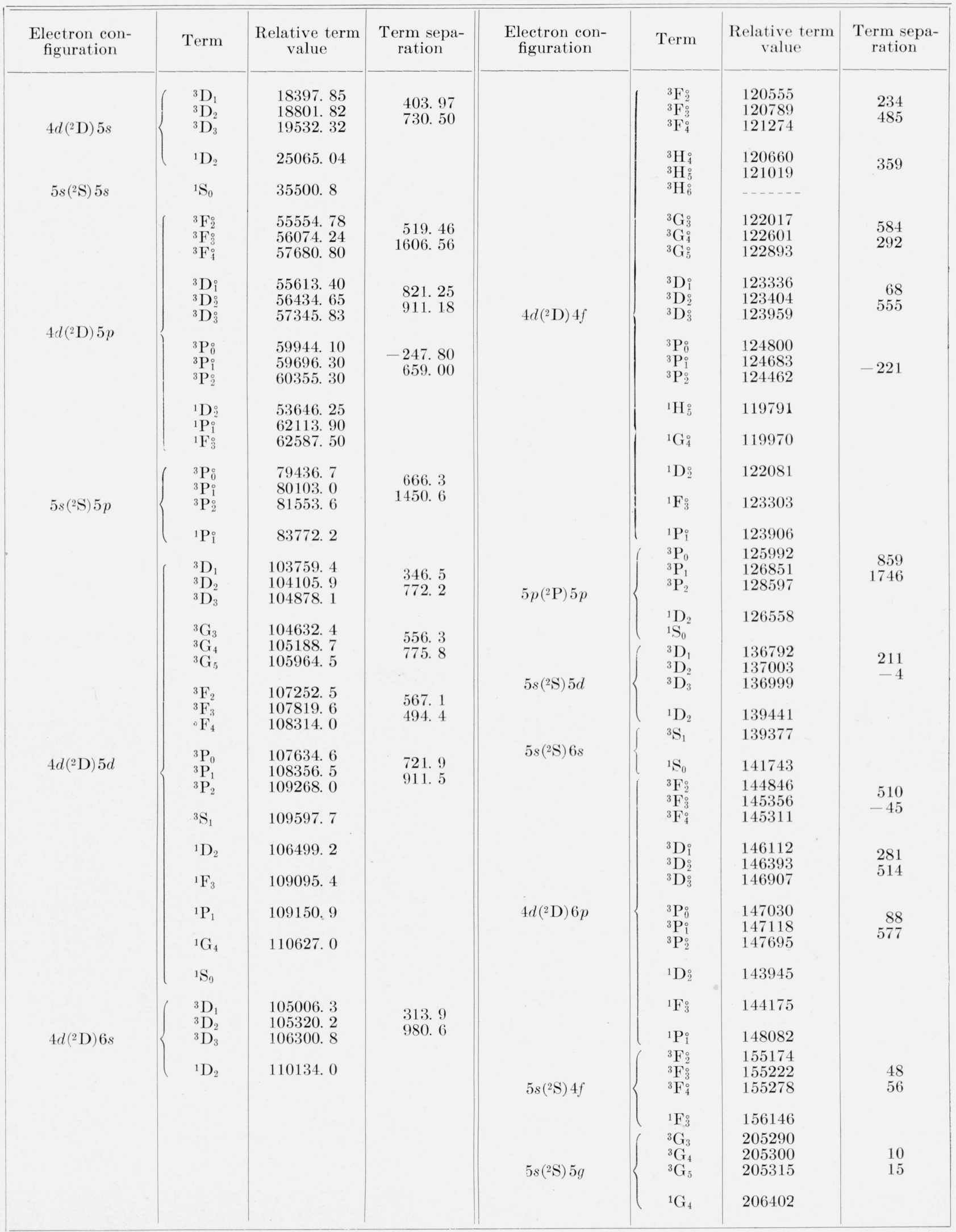


TABLE 6. Classified lines of ' $\mathrm{Hr}$ III

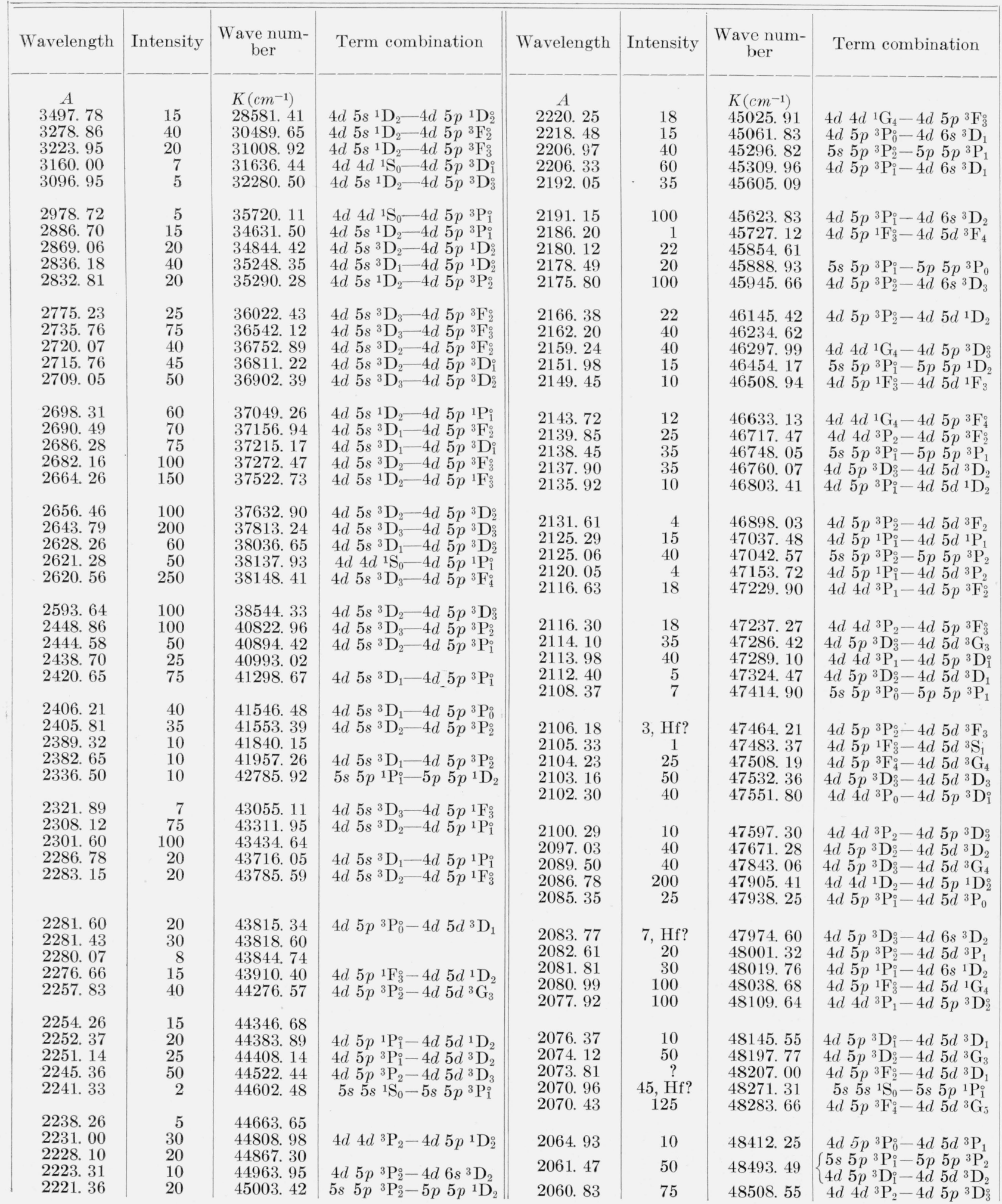


TABLE 6. Classified lines of Zr III-Continued

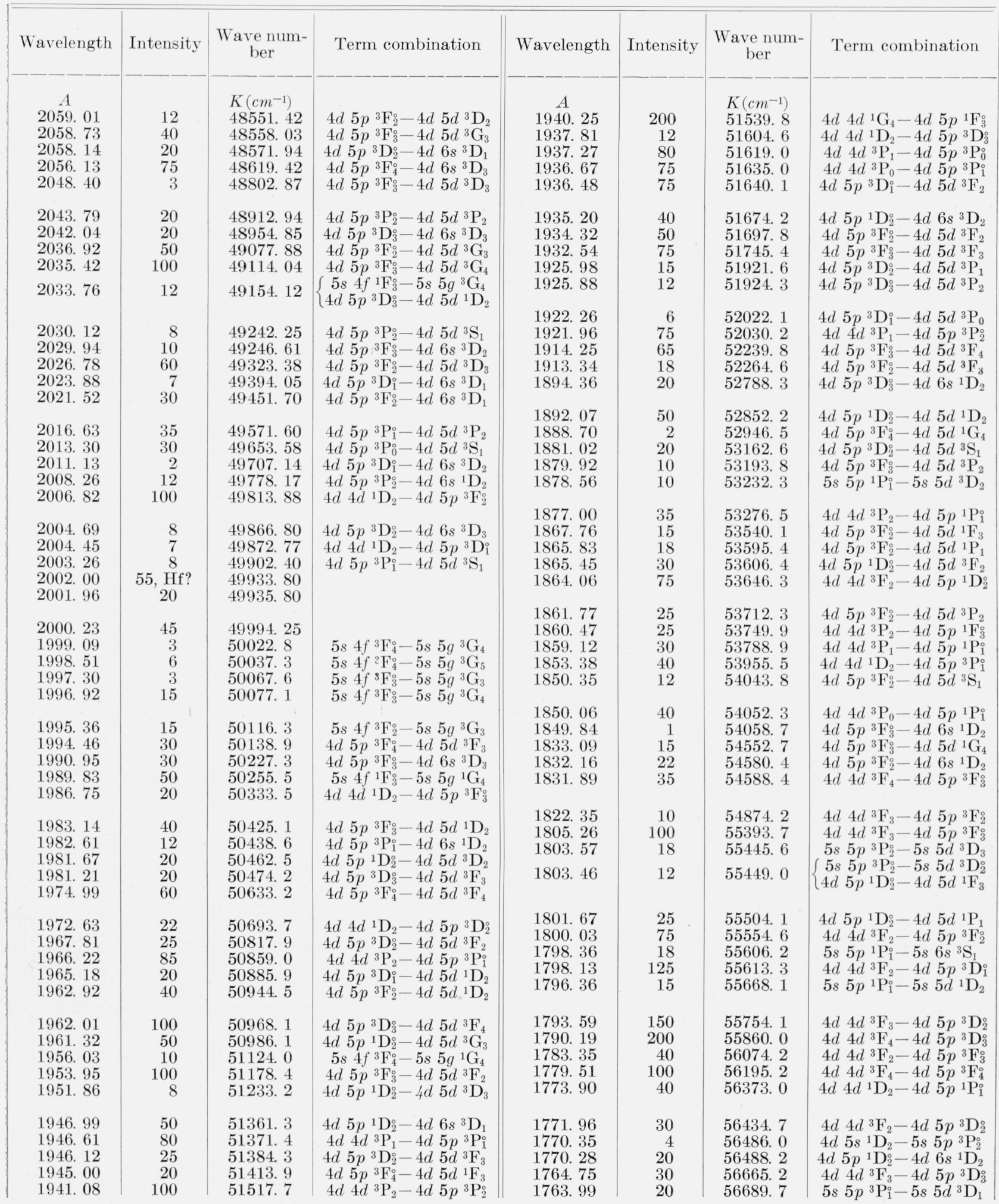


TABLE 6. Classified lines of Zr III-Continued

\begin{tabular}{|c|c|c|c|c|c|c|c|}
\hline Wavelength & Intensity & $\begin{array}{l}\text { Wave num- } \\
\text { ber }\end{array}$ & Term combination & Wavelength & Intensity & $\begin{array}{l}\text { Wave num- } \\
\text { ber }\end{array}$ & Term combination \\
\hline $\begin{array}{c}A \\
1759.12 \\
1757.48 \\
1754.38 \\
1743.55 \\
1729.38\end{array}$ & $\begin{array}{r}35 \\
20 \\
40 \\
20 \\
6\end{array}$ & $\begin{array}{l}K\left(\mathrm{~cm}^{-1}\right) \\
56846.6 \\
56899.7 \\
57000.2 \\
57354.2 \\
57824.2\end{array}$ & $\begin{array}{l}4 d \quad 4 d d^{1} \mathrm{D}_{2}-4 d \quad 5 p p^{1} \mathrm{~F}_{3}^{\circ} \\
5 s \quad 5 p p^{3} \mathrm{P}_{1}-5 s \quad 5 d d^{3} \mathrm{D}_{2} \\
4 d \quad 4 d d^{3} \mathrm{~F}_{3}-4 d 5 p p^{3} \mathrm{~F}_{4}^{\circ} \\
5 s \quad 5 p{ }^{3} \mathrm{P}_{0}^{\circ}-5 s \quad 5 d{ }^{3} \mathrm{D}_{1} \\
5 s \quad 5 p p^{3} \mathrm{P}_{2}^{\circ}-5 s \quad 6 s^{3} \mathrm{~S}_{1}\end{array}$ & $\begin{array}{c}A \\
919.59 \\
918.06 \\
912.28 \\
911.22 \\
909.32\end{array}$ & $\begin{array}{r}25 \\
20 \\
3 \\
1 \\
8\end{array}$ & $\begin{array}{l}K\left(\mathrm{~cm}^{-1}\right) \\
108744 \\
108925 \\
109616 \\
109743 \\
109972\end{array}$ & 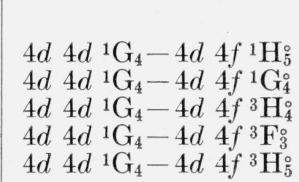 \\
\hline $\begin{array}{l}1727.46 \\
1725.03 \\
1703.36 \\
1687.14 \\
1675.75\end{array}$ & $\begin{array}{r}3 \\
40 \\
40 \\
3 \\
35\end{array}$ & $\begin{array}{l}57888.5 \\
57970.0 \\
58707.5 \\
59271.9 \\
59674.8\end{array}$ & $\begin{array}{l}5 s 5 p{ }^{3} \mathrm{P}_{2}^{\circ}-5 s 5 d{ }^{1} \mathrm{D}_{2} \\
5 s 5 p{ }^{1} \mathrm{P}_{1}^{\circ}-5 s 6 s{ }^{1} \mathrm{~S}_{0} \\
4 d 5 s{ }^{1} \mathrm{D}_{2}-5 s 5 p{ }^{1} \mathrm{P}_{1} \\
5 s 5 p{ }^{3} \mathrm{P}_{1}^{\circ}-5 s 6 s{ }^{3} \mathrm{~S}_{1} \\
4 d \quad 4 d{ }^{3} \mathrm{~F}_{3}-4 d 5 p{ }^{3} \mathrm{P}_{2}^{\circ}\end{array}$ & $\begin{array}{l}907.18 \\
901.15 \\
\text { 896. } 45 \\
\text { 894. } 08 \\
\text { 893. } 22\end{array}$ & $\begin{array}{r}15 \\
2 \\
1 \\
2 \\
4\end{array}$ & $\begin{array}{l}110232 \\
110969 \\
111551 \\
111847 \\
111954\end{array}$ & $\begin{array}{l}4 d \quad 4 d{ }^{1} \mathrm{G}_{4}-4 d \quad 4 f^{3} \mathrm{~F}_{4}^{\circ} \\
4 d \quad 4 d d^{1} \mathrm{G}_{4}-4 d \quad 4 f^{3} \mathrm{G}_{3}^{\circ} \\
4 d \quad 4 d d^{1} \mathrm{G}_{4}-4 d \quad 4 f^{3} \mathrm{G}_{4}^{\circ} \\
4 d \quad 4 d^{1}{ }^{1} \mathrm{G}_{4}-4 d \quad 4 f^{3} \mathrm{G}_{5}^{\circ} \\
4 d d d^{3} \mathrm{P}_{2}-4 d \quad 4 f^{3} \mathrm{~F}_{3}^{\circ}\end{array}$ \\
\hline $\begin{array}{l}\text { 1675. } 06 \\
1672.35 \\
1668.35 \\
1638.33 \\
1636.61\end{array}$ & $\begin{array}{r}35 \\
15 \\
8 \\
50 \\
20\end{array}$ & $\begin{array}{l}59699.4 \\
59796.1 \\
59939.5 \\
61037.8 \\
61101.9\end{array}$ & $\begin{array}{l}4 d 4 d{ }^{3} \mathrm{~F}_{2}-4 d 5 p^{3} \mathrm{P}_{1} \\
4 d 4 d{ }^{1} \mathrm{~S}_{0}-5 s 5 p{ }^{1} \mathrm{P}_{1}^{\circ} \\
5 s 5 p{ }^{3} \mathrm{P}_{0}^{\circ}-5 s 6 s^{3} \mathrm{~S}_{1} \\
4 d 5 s^{3} \mathrm{D}_{1}-5 s 5 p^{3} \mathrm{P}_{0}^{\circ} \\
4 d 4 d^{3} \mathrm{~F}_{4}-4 d 5 p{ }^{1} \mathrm{~F}_{3}^{\circ}\end{array}$ & $\begin{array}{l}\text { 891. } 00 \\
890.78 \\
885.66 \\
883.52 \\
87904\end{array}$ & $\begin{array}{r}1 \\
6 \\
15 \\
2 \\
5\end{array}$ & $\begin{array}{l}112233 \\
112261 \\
112910 \\
113184 \\
113760\end{array}$ & $\begin{array}{l}4 d 4 d d^{3} \mathrm{P}_{1}-4 d 4 f^{3} \mathrm{~F}_{2}^{\circ} \\
4 d 4 d^{1} \mathrm{G}_{4}-4 d \quad 4 f{ }^{1} \mathrm{~F}_{3}^{\circ} \\
4 d 4 d d^{1} \mathrm{G}_{4}-4 d \quad 4 f^{3} \mathrm{D}_{3}^{\circ} \\
4 d 4 d^{3} \mathrm{P}_{2}-4 d \quad 4 f^{3} \mathrm{G}_{3}^{\circ} \\
4 d \quad 4 d^{3} \mathrm{P}_{1}-4 d 4 f^{1} \mathrm{D}_{2}^{\circ}\end{array}$ \\
\hline $\begin{array}{l}\text { 1631. } 31 \\
1628.04 \\
1622.23 \\
1620.62 \\
1615.33\end{array}$ & $\begin{array}{r}75 \\
15 \\
1 \\
50 \\
15\end{array}$ & $\begin{array}{l}61300.4 \\
61423.6 \\
61643.5 \\
61704.8 \\
61906.8\end{array}$ & $\begin{array}{l}4 d 5 s^{3} \mathrm{D}_{2}-5 s 5 p{ }^{3} \mathrm{P}_{1} \\
4 d 5 p{ }^{1} \mathrm{P}_{1}-5 p 5 p \\
5 s 5{ }^{1} \mathrm{~S}_{0} \\
{ }^{3} \mathrm{P}_{1}^{\circ}-5 s 6 s{ }^{1} \mathrm{~S}_{0} \\
4 d 5 s^{3} \mathrm{D}_{1}-5 s 5 p{ }^{3} \mathrm{P}_{1}^{\circ} \\
4 d 4 d^{3} \mathrm{~F}_{3}-4 d 5 p{ }^{1} \mathrm{~F}_{3}^{\circ}\end{array}$ & $\begin{array}{l}873.61 \\
873.36 \\
872.76 \\
870.93 \\
869.47\end{array}$ & $\begin{array}{r}18 \\
6 \\
12 \\
5 \\
12\end{array}$ & $\begin{array}{l}114468 \\
114500 \\
114579 \\
114820 \\
115013\end{array}$ & 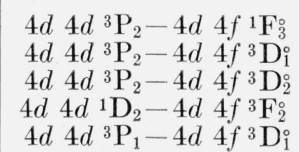 \\
\hline $\begin{array}{l}1612.38 \\
1609.94 \\
1593.59 \\
1583.39 \\
1566.35\end{array}$ & $\begin{array}{r}100 \\
20 \\
40 \\
20 \\
1\end{array}$ & $\begin{array}{l}\text { 62020. } 1 \\
62114.1 \\
62751.4 \\
63155.6 \\
63842.7\end{array}$ & $\begin{array}{l}4 d 5 s^{3} \mathrm{D}_{3}-5 s 5 p{ }^{3} \mathrm{P}_{2}^{\circ} \\
4 d 4 d{ }^{3} \mathrm{~F}_{2}-4 d 5 p{ }^{1} \mathrm{P}_{1}^{\circ} \\
4 d 5 s{ }^{3} \mathrm{D}_{2}-5 s 5 p{ }^{3} \mathrm{P}_{2}^{\circ} \\
4 d 5 s^{3} \mathrm{D}_{1}-5 s 5 p{ }^{3} \mathrm{P}_{2}^{\circ} \\
4 d 5 p^{3} \mathrm{P}_{1}^{\circ}-5 p 5 p{ }^{1} \mathrm{~S}_{0}\end{array}$ & $\begin{array}{l}869.18 \\
868.99 \\
868.64 \\
867.50 \\
865.19\end{array}$ & $\begin{array}{r}2 \\
25 \\
25 \\
20 \\
4\end{array}$ & $\begin{array}{l}115043 \\
115076 \\
115122 \\
115274 \\
115582\end{array}$ & $\begin{array}{lll}4 d & 4 d d^{1} \mathrm{D}_{2}-4 d & 4 f^{3} \mathrm{~F}_{3}^{\circ} \\
4 d & 4 d d^{3} \mathrm{P}_{1}-4 d & 4 f^{3} \mathrm{D}_{2}^{\circ} \\
4 d & 4 d d^{3} \mathrm{P}_{2}-4 d & 4 f^{3} \mathrm{D}_{3}^{\circ} \\
4 d & 4 d d^{3} \mathrm{P}_{0}-4 d & 4 f^{3} \mathrm{D}_{1}^{\circ} \\
4 d & 4 d^{3} \mathrm{P}_{1}-4 d & 4 f^{1} \mathrm{P}_{1}^{\circ}\end{array}$ \\
\hline $\begin{array}{l}1565.45 \\
1563.24 \\
1539.07 \\
1514.93 \\
1510.52\end{array}$ & $\begin{array}{r}10 \\
25 \\
3 \\
8 \\
10\end{array}$ & $\begin{array}{l}63879.4 \\
63969.7 \\
64974.3 \\
66009.6 \\
66202.4\end{array}$ & $\begin{array}{l}4 d 5 p{ }^{1} \mathrm{P}_{1}^{\circ}-5 p 5 p{ }^{3} \mathrm{P}_{0} \\
4 d 5 p{ }^{1} \mathrm{~F}_{3}^{\circ}-5 p 5 p{ }^{1} \mathrm{D}_{2} \\
4 d 5 s{ }^{3} \mathrm{D}_{2}-5 s 5 p{ }^{1} \mathrm{P}_{1} \\
4 d 5 p{ }^{1} \mathrm{~F}_{3}^{\circ}-5 p 5 p{ }^{3} \mathrm{P}_{2} \\
4 d 5 p^{3} \mathrm{P}_{2}^{\circ}-5 p 5 p{ }^{1} \mathrm{D}_{2}\end{array}$ & $\begin{array}{l}\text { 864. } 86 \\
\text { 863. } 23 \\
\text { 860. } 04\end{array}$ & $\begin{array}{r}30 \\
15 \\
7\end{array}$ & $\begin{array}{l}115626 \\
115844 \\
116274\end{array}$ & 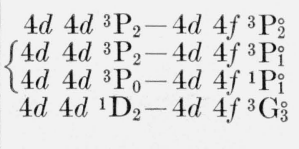 \\
\hline $\begin{array}{l}1508.38 \\
1503.87 \\
1495.66 \\
1494.67 \\
1489.13\end{array}$ & $\begin{array}{r}5 \\
6 \\
2 \\
10 \\
12\end{array}$ & $\begin{array}{l}\text { 66296. } 3 \\
66495.1 \\
66860.1 \\
66904.4 \\
67153.3\end{array}$ & $\begin{array}{l}4 d 5 p p^{3} \mathrm{P}_{1}^{\circ}-5 p 5 p p^{3} \mathrm{P}_{0} \\
4 d 5 p p^{3} \mathrm{P}_{2}^{\circ}-5 p 5 p p^{3} \mathrm{P}_{1} \\
4 d 5 p{ }^{3} \mathrm{P}_{1}^{\circ}-5 p 5 p p^{1} \mathrm{D}_{2} \\
4 d 5 p{ }^{3} \mathrm{P}_{0}^{\circ}-5 p 5 p p p^{3} \mathrm{P}_{1} \\
4 d 5 p{ }^{3} \mathrm{P}_{1}^{\circ}-5 p 5 p p^{3} \mathrm{P}_{1}\end{array}$ & $\begin{array}{l}\text { 859. } 56 \\
859.42 \\
858.56 \\
857.49 \\
850.61\end{array}$ & $\begin{array}{r}25 \\
12 \\
4 \\
4 \\
30\end{array}$ & $\begin{array}{l}116339 \\
116358 \\
116474 \\
116619 \\
117563\end{array}$ & $\begin{array}{l}4 d 4 d d^{1} \mathrm{D}_{2}-4 d \quad 4 f^{1} \mathrm{D}_{2}^{\circ} \\
4 d 4 d d^{3} \mathrm{P}_{1}-4 d \quad 4 f^{3} \mathrm{P}_{1}^{\circ} \\
4 d 4 d^{3} \mathrm{P}_{1}-4 d \quad 4 f^{3} \mathrm{P}_{0}^{\circ} \\
4 d 4 d^{3} \mathrm{P}_{0}-4 d \quad 4 f^{3} \mathrm{P}_{1}^{\circ} \\
4 d 4 d^{1} \mathrm{D}_{2}-4 d \quad 4 f^{1} \mathrm{~F}_{3}^{\circ}\end{array}$ \\
\hline $\begin{array}{l}1465.44 \\
1451.40 \\
1444.88 \\
1426.11 \\
1420.87\end{array}$ & $\begin{array}{r}25 \\
8 \\
20 \\
5 \\
25\end{array}$ & $\begin{array}{l}\text { 68238. } 9 \\
68899.0 \\
69209.9 \\
70120.8 \\
70379.4\end{array}$ & $\begin{array}{l}4 d 5 p^{3} \mathrm{P}_{2}^{\circ}-5 p 5 p{ }^{3} \mathrm{P}_{2} \\
4 d 5 p{ }^{3} \mathrm{P}_{1}^{\circ}-5 p 5 p{ }^{3} \mathrm{P}_{2} \\
4 d 5 p{ }^{3} \mathrm{D}_{3}^{\circ}-5 p 5 p{ }^{1} \mathrm{D}_{2} \\
4 d 5 p^{3} \mathrm{D}_{2}^{\circ}-5 p 5 p{ }^{1} \mathrm{D}_{2} \\
4 d 5 p^{3} \mathrm{D}_{1}^{\circ}-5 p 5 p{ }^{3} \mathrm{P}_{0}\end{array}$ & $\begin{array}{l}849.92 \\
845.89 \\
845.26 \\
844.00 \\
842.33\end{array}$ & $\begin{array}{r}3 \\
18 \\
8 \\
3 \\
2\end{array}$ & $\begin{array}{l}117658 \\
118219 \\
118307 \\
118483 \\
118718\end{array}$ & $\begin{array}{lll}4 d & 4 d d^{1} \mathrm{D}_{2}-4 d & 4 f^{3} \mathrm{D}_{2}^{\circ} \\
4 d & 4 d d^{1}{ }^{1} \mathrm{D}_{2}-4 d & 4 f^{3} \mathrm{D}_{3}^{\circ} \\
4 d & 4 d^{3} \mathrm{~F}_{4}-4 d & 4 f^{1}{ }^{1} \mathrm{H}_{5}^{\circ} \\
4 d & 4 d^{3}{ }^{3} \mathrm{~F}_{4}-4 d & 4 f^{1} \mathrm{G}_{4}^{\circ} \\
4 d & 4 d d^{1} \mathrm{D}_{2}-4 d & 4 f^{3} \mathrm{P}_{2}^{\circ}\end{array}$ \\
\hline $\begin{array}{l}1420.12 \\
1418.81 \\
1408.40 \\
1403.74 \\
1403.48\end{array}$ & $\begin{array}{r}35 \\
6 \\
15 \\
20 \\
40\end{array}$ & $\begin{array}{l}70416.6 \\
70481.6 \\
71002.6 \\
71238.3 \\
71251.5\end{array}$ & $\begin{array}{l}4 d 5 p^{3} \mathrm{D}_{2}^{\circ}-5 p 5 p^{3} \mathrm{P}_{1} \\
4 d 5 p^{3} \mathrm{~F}_{3}^{\circ}-5 p 5 p{ }^{1} \mathrm{D}_{2} \\
4 d 5 p^{3} \mathrm{~F}_{2}^{\circ}-5 p 5 p{ }^{1} \mathrm{D}_{2} \\
4 d 5 p{ }^{3} \mathrm{D}_{1}^{\circ}-5 p 5 p{ }^{3} \mathrm{P}_{1} \\
4 d 5 p^{3} \mathrm{D}_{3}^{\circ}-5 p 5 p^{3} \mathrm{P}_{2}\end{array}$ & $\begin{array}{l}840.77 \\
839.55 \\
839.12 \\
838.31 \\
838.20\end{array}$ & $\begin{array}{r}1 \\
12 \\
5 \\
4 \\
7\end{array}$ & $\begin{array}{l}118939 \\
119111 \\
119172 \\
119288 \\
119303\end{array}$ & $\begin{array}{l}4 d 4 d^{1} \mathrm{D}_{2}-4 d \quad 4 f^{3} \mathrm{P}_{1}^{\circ} \\
4 d \quad 4 d^{3} \mathrm{~F}_{3}-4 d \quad 4 f^{1} \mathrm{H}_{5}^{\circ} \\
4 d \quad 4 d^{3} \mathrm{~F}_{4}-4 d \quad 4 f^{3} \mathrm{H}_{4}^{\circ} \\
4 d 4 d^{3} \mathrm{~F}_{3}-4 d \quad 4 f^{1} \mathrm{G}_{4}^{\circ} \\
4 d 4 d^{3} \mathrm{~F}_{4}-4 d \quad 4 f^{3} \mathrm{~F}_{3}^{\circ}\end{array}$ \\
\hline $\begin{array}{l}1402.62 \\
1393.20 \\
1385.75 \\
1378.93 \\
1375.13\end{array}$ & $\begin{array}{r}4 \\
6 \\
18 \\
30 \\
25\end{array}$ & $\begin{array}{l}71295.1 \\
71777.2 \\
72163.1 \\
72520.0 \\
72720.4\end{array}$ & $\begin{array}{l}4 d 5 p^{3} \mathrm{~F}_{2}^{\circ}-5 p 5 p^{3} \mathrm{P}_{1} \\
4 d 4 d^{3} \mathrm{P}_{1}-5 s 5 p{ }^{3} \mathrm{P}_{1} \\
4 d 5 p^{3} \mathrm{D}_{2}^{\circ}-5 p 5 p{ }^{3} \mathrm{P}_{2} \\
4 d 5 p{ }^{3} \mathrm{~F}_{3}^{\circ}-5 p 5 p{ }^{3} \mathrm{P}_{2} \\
4 d 4 d^{3} \mathrm{P}_{2}-5 s 5 p^{3} \mathrm{P}_{2}^{\circ}\end{array}$ & $\begin{array}{l}836.60 \\
834.85 \\
834.19 \\
833.51 \\
832.59\end{array}$ & $\begin{array}{r}18 \\
20 \\
8 \\
10 \\
15\end{array}$ & $\begin{array}{l}119531 \\
119782 \\
119877 \\
119975 \\
120107\end{array}$ & $\begin{array}{l}4 d \quad 4 d^{3} \mathrm{~F}_{4}-4 d \quad 4 f^{3} \mathrm{H}_{5}^{\circ} \\
4 d \quad 4 d^{3} \mathrm{~F}_{4}-4 d \quad 4 f^{3} \mathrm{~F}_{4}^{\circ} \\
4 d \quad 4 d^{3} \mathrm{~F}_{3}-4 d \quad 4 f^{3} \mathrm{~F}_{2}^{\circ} \\
4 d \quad 4 d^{3} \mathrm{~F}_{3}-4 d \quad 4 f^{3} \mathrm{H}_{4}^{\circ} \\
4 d \quad 4 d^{3} \mathrm{~F}_{3}-4 d \quad 4 f^{3} \mathrm{~F}_{3}^{\circ}\end{array}$ \\
\hline $\begin{array}{l}1371.50 \\
1369.07 \\
1320.81 \\
1281.53 \\
1000.72\end{array}$ & $\begin{array}{r}20 \\
4 \\
30 \\
1 \\
6\end{array}$ & $\begin{array}{l}\text { 72912. } 9 \\
73042.3 \\
75711.1 \\
78031.7 \\
99928\end{array}$ & 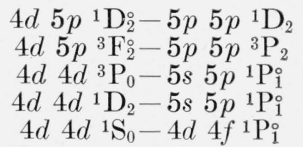 & $\begin{array}{l}829.50 \\
829.24 \\
827.90 \\
825.65 \\
824.16\end{array}$ & $\begin{array}{l}25 \\
15 \\
20 \\
18 \\
20\end{array}$ & $\begin{array}{l}120555 \\
120592 \\
120788 \\
121117 \\
121336\end{array}$ & $\begin{array}{l}4 d \quad 4 d^{3} \mathrm{~F}_{2}-4 d \quad 4 f^{3} \mathrm{~F}_{2}^{\circ} \\
4 d \quad 4 d d^{3} \mathrm{~F}_{3}-4 d \quad 4 f^{3} \mathrm{~F}_{4}^{\circ} \\
4 d \quad 4 d d^{3} \mathrm{~F}_{2}-4 d \quad 4 f^{3} \mathrm{~F}_{3}^{\circ} \\
4 d \quad 4 d^{3} \mathrm{~F}_{4}-4 d \quad 4 f^{3} \mathrm{G}_{4}^{\circ} \\
4 d \quad 4 d^{3} \mathrm{~F}_{3}-4 d \quad 4 f^{3} \mathrm{G}_{3}^{\circ}\end{array}$ \\
\hline
\end{tabular}


TABLE 6. Classified lines of $\mathrm{Zr} \mathrm{III-Continued}$

\begin{tabular}{|c|c|c|c|c|c|c|c|}
\hline Wavelength & Intensity & $\begin{array}{l}\text { Wave num- } \\
\text { ber }\end{array}$ & Term combination & Wavelength & Intensity & $\begin{array}{l}\text { Wave num- } \\
\text { ber }\end{array}$ & Term combination \\
\hline$A$ & & $K\left(\mathrm{~cm}^{-1}\right)$ & & $A$ & & $K\left(\mathrm{~cm}^{-1}\right)$ & \\
\hline 823.69 & 35 & 121405 & $4 d 4 d^{3} \mathrm{~F}_{4}-4 d 4 f^{3} \mathrm{G}_{5}^{\circ}$ & 719.16 & 1 & 139051 & $4 d 4 d{ }^{3} \mathrm{P}_{0}-4 d 6 p^{3} \mathrm{P}_{1}^{\circ}$ \\
\hline 820.91 & 8 & 121816 & $4 d 4 d^{3} \mathrm{~F}_{4}-4 d 4 f^{1} \mathrm{~F}_{3}^{\circ}$ & 717.53 & 3 & 139367 & $4 d 4 d^{3} \mathrm{P}_{1}-4 d 6 p^{3} \mathrm{P}_{2}^{0}$ \\
\hline 820,21 & 30 & 121920 & $4 d 4 d^{3} \mathrm{~F}_{3}-4 d 4 f^{3} \mathrm{G}_{4}^{\circ}$ & 716.28 & 7 & 139610 & $4 d 4 d^{1} \mathrm{D}_{2}-4 d 6 p{ }^{3} \mathrm{~F}_{3}^{\circ}$ \\
\hline 819.59 & 25 & 122012 & $4 d 4 d^{3} \mathrm{~F}_{2}-4 d 4 f^{3} \mathrm{G}_{3}^{\circ}$ & 715.53 & 7 & 139756 & $4 d 4 d{ }^{3} \mathrm{P}_{1}-4 d 6 p{ }^{1} \mathrm{P}_{1}^{\circ}$ \\
\hline 819.14 & 1 & 122079 & $4 d 4 d^{3} \mathrm{~F}_{2}-4 d 4 f^{1} \mathrm{D}_{2}^{\circ}$ & 714.18 & 6 & 140020 & $4 d 4 d{ }^{3} \mathrm{P}_{0}-4 d 6 p{ }^{1} \mathrm{P}_{1}^{\circ}$ \\
\hline 816.53 & 6 & 122470 & $4 d 4 d^{3} \mathrm{~F}_{4}-4 d 4 f^{3} \mathrm{D}_{3}^{\circ}$ & 710.98 & 10 & 140651 & $4 d 4 d^{1} \mathrm{D}_{2}-4 d 6 p^{3} \mathrm{D}_{2}^{\circ}$ \\
\hline 815.53 & 5 & 122620 & $4 d 4 d^{3} \mathrm{~F}_{3}-4 d 4 f^{1} \mathrm{~F}_{3}^{\circ}$ & 708. 40 & 8 & 141163 & $4 d 4 d^{1} \mathrm{D}_{2}-4 d 6 p^{3} \mathrm{D}_{3}^{0}$ \\
\hline 814.89 & 12 & 122716 & $4 d 4 d^{3} \mathrm{~F}_{3}-4 d 4 f^{3} \mathrm{D}_{2}^{\circ}$ & 700.80 & 2 & 142694 & $4 d 4 d^{3} \mathrm{~F}_{4}-4 d 6 p^{1} \mathrm{~F}_{3}^{\circ}$ \\
\hline 811.00 & 6 & 123305 & $4 d 4 d^{3} \mathrm{~F}_{2}-4 d 4 f^{1} \mathrm{~F}_{3}^{\circ}$ & 696.91 & 4 & 143491 & $4 d 4 d^{3} \mathrm{~F}_{3}-4 d 6 p^{1} \mathrm{~F}_{3}^{\circ}$ \\
\hline 810.80 & 6 & 123335 & $4 d 4 d{ }^{3} \mathrm{~F}_{2}-4 d 4 f^{3} \mathrm{D}_{1}^{\circ}$ & 695. 29 & 12 & 143825 & $4 d 4 d^{3} \mathrm{~F}_{4}-4 d 6 p{ }^{3} \mathrm{~F}_{4}^{\circ}$ \\
\hline 807.88 & 2 & 123781 & $4 d 4 d^{3} \mathrm{~F}_{3}-4 d 4 f^{3} \mathrm{P}_{2}^{\circ}$ & 695.06 & 10 & 143872 & $4 d 4 d^{3} \mathrm{~F}_{4}-4 d 6 p^{3} \mathrm{~F}_{3}^{\circ}$ \\
\hline 802.00 & 4 & 124688 & $4 d 4 d^{3} \mathrm{~F}_{2}-4 d 4 f^{3} \mathrm{P}_{1}$ & 694.69 & 8 & 143949 & $4 d 4 d^{3} \mathrm{~F}_{2}-4 d 6 p{ }^{1} \mathrm{D}_{2}^{\circ}$ \\
\hline 762.90 & 20 & 131079 & $4 d 5 s^{1} \mathrm{D}_{2}-5 s \quad 4 f^{1} \mathrm{~F}_{3}^{\circ}$ & 693.65 & 2 & 144165 & $4 d 4 d^{3} \mathrm{~F}_{3}-4 d 6 p{ }^{3} \mathrm{~F}_{2}^{\circ}$ \\
\hline 751.16 & 5 & 133127 & $4 d 4 d^{1} \mathrm{G}_{4}-4 d 6 p^{1} \mathrm{~F}_{3}^{\circ}$ & 691.42 & 15 & 144630 & $4 d 4 d^{3} \mathrm{~F}_{3}-4 d 6 p{ }^{3} \mathrm{~F}_{4}^{\circ}$ \\
\hline 744.58 & 4 & 134304 & $4 d 4 d^{1} \mathrm{G}_{4}-4 d 6 p^{3} \mathrm{~F}_{3}^{\circ}$ & 691. 19 & 10 & 144678 & $4 d 4 d^{3} \mathrm{~F}_{3}-4 d 6 p^{3} \mathrm{~F}_{3}^{\circ}$ \\
\hline 736.97 & 4 & 135691 & $4 d 5 s^{3} \mathrm{D}_{3}-5 s \quad 4 f^{3} \mathrm{~F}_{3}^{\circ}$ & 690.39 & 50 & 144846 & $4 d 4 d^{3} \mathrm{~F}_{2}-4 d 6 p^{3} \mathrm{~F}_{2}^{\circ}$ \\
\hline 736.67 & 20 & 135746 & $4 d 5 s^{3} \mathrm{D}_{3}-5 s 4 f^{3} \mathrm{~F}_{4}^{\circ}$ & 687.95 & 12 & 145359 & $4 d 4 d^{3} \mathrm{~F}_{2}^{2}-4 d 6 p^{3} \mathrm{~F}_{3}^{\circ}$ \\
\hline 733. 29 & 4 & 136372 & $4 d 5 s^{3} \mathrm{D}_{2}-5 s 4 f^{3} \mathrm{~F}_{2}^{\circ}$ & 687.64 & 25 & 145425 & $4 d 4 d^{3} \mathrm{~F}_{4}-4 d 6 p^{3} \mathrm{D}_{3}^{\circ}$ \\
\hline 733. 03 & 18 & 136420 & $4 d 5 s^{3} \mathrm{D}_{2}-5 s 4 f^{3} \mathrm{~F}_{3}^{\circ}$ & 686. 28 & 4 & 145713 & $4 d 4 d{ }^{3} \mathrm{~F}_{3}-4 d 6 p^{3} \mathrm{D}_{2}^{\circ}$ \\
\hline 731.98 & 2 & 136616 & $4 d 5 s^{3} \mathrm{D}_{3}-5 s \quad 4 f^{1} \mathrm{~F}_{3}^{\circ}$ & 684.38 & 2 & 146118 & $4 d 4 d d^{3} \mathrm{~F}_{2}-4 d 6 p{ }^{3} \mathrm{D}_{1}^{\circ}$ \\
\hline 731. 12 & 10 & 136776 & $4 d 5 s^{3} \mathrm{D}_{1}-5 s 4 f^{3} \mathrm{~F}_{2}^{\circ}$ & 683.09 & 5 & 146394 & $4 d 4 d^{3} \mathrm{~F}_{2}-4 d 6 p^{3} \mathrm{D}_{2}$ \\
\hline 726.99 & 5 & 137554 & $4 d 4 d^{3} \mathrm{P}_{2}-4 d 6 p{ }^{3} \mathrm{D}_{2}^{\circ}$ & 680.69 & 3 & 146910 & $4 d 4 d^{3} \mathrm{~F}_{2}-4 d 6 p{ }^{3} \mathrm{D}_{3}^{\circ}$ \\
\hline 724.40 & 12 & 138045 & $4 d 4 d^{3} \mathrm{P}_{0}-4 d 6 p^{3} \mathrm{D}_{1}^{\circ}$ & & & & \\
\hline 724.30 & 15 & 138064 & 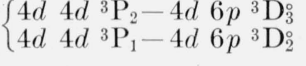 & & & & \\
\hline 723.59 & 15 & 138200 & $4 d 4 d^{1} \mathrm{D}_{2}-4 d 6 p^{1} \mathrm{D}_{2}^{\circ}$ & & & & \\
\hline 723. 13 & 4 & 138288 & $4 d 4 d{ }^{3} \mathrm{P}_{2}-4 d 6 p{ }^{3} \mathrm{P}_{1}{ }^{2}$ & & & & \\
\hline 720.96 & 2 & 138704 & $4 d 4 d^{3} \mathrm{P}_{1}-4 d 6 p^{3} \mathrm{P}_{0}^{0}$ & & & & \\
\hline $\begin{array}{l}720.50 \\
720.15\end{array}$ & $\begin{array}{l}5 \\
3\end{array}$ & 138792 & $4 d 4 d^{3} \mathrm{P}_{1}-4 d 6{ }^{3}{ }^{3} \mathrm{P}_{1}$ & & & & \\
\hline 720.15 & 3 & 138860 & $4 d 4 d^{3} \mathrm{P}_{2}-4 d 6 p^{3} \mathrm{P}_{2}^{\circ}$ & & & & \\
\hline
\end{tabular}

TABLE 7. Zeeman patterns of Zr III lines

$A=$ shaded outward $/ \| \backslash B=$ shaded inward $[\backslash / \mid$.

\begin{tabular}{|c|c|c|c|}
\hline Wavelengths & Term combinations & Observed Zeeman patterns & Derived $g$-values \\
\hline$A$ & & & \\
\hline 3497.78 & $5 s^{1} \mathrm{D}_{2}-5 p{ }^{1} \mathrm{D}_{2}^{\circ}$ & $(0.128) 0.993$ & 1. $070 ; 0.914$ \\
\hline 2836. 18 & $5 s^{3} \mathrm{D}_{1}-5 p^{1} \mathrm{D}_{2}$ & $(0.000 .0 .422)$ & $0.495 ; 0.914$ \\
\hline 2735.76 & $5 s^{3} \mathrm{D}_{3}-5 p^{3} \mathrm{~F}_{3}^{\circ}$ & $(0.438, \mathbf{0 . 6 8 4}) 0.878, \mathbf{1 . 1 0 4}, 1.345,1.552$ & 1. $345 ; 1.104$ \\
\hline 2715. 76 & $5 s^{3} \mathrm{D}_{2}-5 p{ }^{3} \mathrm{D}_{1}^{\circ}$ & $(0.000) 1.824$ & $1.162 ; 0.500$ \\
\hline 2698. 31 & $5 s^{1} \mathrm{D}_{2}-5 p{ }^{1} \mathrm{P}_{1}^{\circ}$ & $(0.000) 0.972$ & 1,$070 ; 1.266$ \\
\hline 2690. 49 & $5 s^{3} \mathrm{D}_{1}-5 p{ }^{3} \mathrm{~F}_{2}^{\circ}$ & $(\mathbf{0 . 0 0 0 ,} 0.281) \quad 0.791, \mathbf{1 . 0 7 4}$ & $0.495 ; 0.792$ \\
\hline 2686. 28 & $5 s^{3} \mathrm{D}_{1}-5 p^{3} \mathrm{D}_{i}^{\circ}$ & $(0.000) 0.506$ & $0.495 ; 0.500$ \\
\hline 2682. 16 & $5 s^{3} \mathrm{D}_{2}-5 p^{3} \mathrm{~F}_{3}^{\circ}$ & $0.000) 1.040 A$ & 1. $162 ; 1.104$ \\
\hline 2664. 26 & $5 s^{1} \mathrm{D}_{2}-5 p^{1} \mathrm{~F}_{3}^{\circ}$ & $(0.000) 1.020$ & $1.070 ; 1.045$ \\
\hline 2656. 46 & $5 s^{3} \mathrm{D}_{2}-5 p^{3} \mathrm{D}_{2}^{\circ}$ & $(0.000) 1.166$ & 1. $162 ; 1.136$ \\
\hline 2643. 79 & $5 s^{3} \mathrm{D}_{3}-5 p{ }^{3} \mathrm{D}_{3}^{\circ}$ & $(0.000) 1.341$ & 1. $345 ; 1.364$ \\
\hline 2628. 26 & $5 s^{3} \mathrm{D}_{1}-5 p^{3} \mathrm{D}_{2}^{\circ}$ & $(\mathbf{0 . 0 0 0}, 0.666) 1.150, \mathbf{1 . 7 8 8}$ & $0.495 ; 1.136$ \\
\hline $\begin{array}{l}2620.56 \\
2593.64\end{array}$ & $5 s^{3} \mathrm{D}_{3}-5 p{ }^{3} \mathrm{~F}_{4}^{\circ}$ & $(0.000) 1.042 \mathrm{~A}$ & 1. $345 ; 1.224$ \\
\hline 2090.04 & $5 s^{\circ} D_{2}$ & (0.000 w) $1.000 \mathrm{D}$ & $1.102,1.004$ \\
\hline
\end{tabular}


TaBle 8. Series terms of $\mathrm{Zr}$ III

\begin{tabular}{|c|c|c|c|c|c|c|}
\hline Terms & $\begin{array}{l}\text { Limits in } \\
\text { Zr IV }\end{array}$ & $\begin{array}{l}\text { Relative } \\
\text { term- } \\
\text { values }\end{array}$ & $\begin{array}{l}\text { Term } \\
\text { separations }\end{array}$ & $\begin{array}{c}\text { Distances } \\
\text { to limits }\end{array}$ & $\begin{array}{l}\text { Distances to } \\
\text { ground state } \\
\text { of } \mathrm{Zr} \text { III }\end{array}$ & $\begin{array}{l}\text { Distances } \\
\text { between } \\
\text { ground states } \\
\text { of } \mathrm{Zr} \text { III-Zr IV }\end{array}$ \\
\hline $\begin{array}{l}4 d 4 d{ }^{1} \mathrm{G}_{4} \\
4 d 5 d^{1} \mathrm{G}_{4}\end{array}$ & $4 d^{2} \mathrm{D}_{21 / 2}$ & $\begin{array}{r}11048 \\
110627\end{array}$ & $\begin{array}{l}c m-1 \\
99579\end{array}$ & $\begin{array}{r}c m-1 \\
192085 \\
92505\end{array}$ & $\begin{array}{l}c m-1 \\
+9798\end{array}$ & 201883 \\
\hline $\begin{array}{l}4 d 4 d d^{3} \mathrm{~F}_{2} \\
4 d 5 d^{3} \mathrm{~F}_{2}\end{array}$ & $4 d{ }^{2} \mathrm{D}_{11 / 2}$ & $\begin{array}{r}0 \\
107252\end{array}$ & 107252 & $\begin{array}{r}203530 \\
96278\end{array}$ & 0 & 203530 \\
\hline $\begin{array}{l}4 d 5 s{ }^{1} \mathrm{D}_{2} \\
4 d 6 s{ }^{1} \mathrm{D}_{2}\end{array}$ & $4 d{ }^{2} \mathrm{D}_{21 / 2}$ & $\begin{array}{r}25065 \\
110134\end{array}$ & 85069 & $\begin{array}{r}169981 \\
84912\end{array}$ & +23815 & 193796 \\
\hline $\begin{array}{l}4 d 5 s{ }^{3} \mathrm{D}_{1} \\
4 d 6 s{ }^{3} \mathrm{D}_{1}\end{array}$ & $4 d{ }^{2} \mathrm{D}_{11 / 2}$ & $\begin{array}{r}18398 \\
105006\end{array}$ & 86608 & $\begin{array}{r}172357 \\
85749\end{array}$ & +18398 & 190755 \\
\hline $\begin{array}{lll}4 d & 5 p & { }^{1} \mathrm{D}_{2}^{\circ} \\
4 d & 6 p & { }^{1} \mathrm{D}_{2}^{\circ}\end{array}$ & $4 d^{2} \mathrm{D}_{21 / 2}$ & $\begin{array}{r}53646 \\
143945\end{array}$ & 90299 & $\begin{array}{r}178028 \\
87725\end{array}$ & +52396 & 230424 a \\
\hline $\begin{array}{l}4 d 5 p^{3} \mathrm{~F}_{2}^{\circ} \\
4 d 6 p^{3} \mathrm{~F}_{2}^{\circ}\end{array}$ & $4 d{ }^{2} \mathrm{D}_{11 / 2}$ & $\begin{array}{r}55555 \\
144846\end{array}$ & 89291 & $\begin{array}{r}176479 \\
87188\end{array}$ & +55555 & $230034^{\text {a }}$ \\
\hline $\begin{array}{lll}5 s & 5 s & { }^{1} \mathbf{S}_{0} \\
5 s & 6 s & { }^{1} \mathbf{S}_{0}\end{array}$ & $5 s{ }^{2} \mathbf{S}_{01 / 2}$ & $\begin{array}{r}35501 \\
141743\end{array}$ & 106242 & $\begin{array}{r}202031 \\
95789\end{array}$ & -2757 & 199274 \\
\hline $\begin{array}{l}5 s \quad 4 d^{1}{ }^{1} \mathrm{D}_{2} \\
5 s \quad 5 d^{1}{ }^{1} \mathrm{D}_{2}\end{array}$ & $5 s{ }^{2} \mathbf{S}_{01 / 2}$ & $\begin{array}{r}25065 \\
139441\end{array}$ & 114376 & $\begin{array}{r}214028 \\
99652\end{array}$ & -13193 & 200835 \\
\hline $\begin{array}{l}5 s \quad 4 d d^{3} \mathrm{D}_{1} \\
5 s \quad 5 d{ }^{3} \mathrm{D}_{1}\end{array}$ & $5 s{ }^{2} \mathbf{S}_{01 / 2}$ & $\begin{array}{r}18398 \\
136792\end{array}$ & 118394 & $\begin{array}{l}219902 \\
101507\end{array}$ & -19860 & 200043 \\
\hline \multicolumn{7}{|c|}{ Mean Zr III-Zr IV = $\mathbf{1 9 8 5 9 0}$} \\
\hline \multicolumn{7}{|c|}{ Ionization potential $=198590 \times 1.2395 \times 10^{-4}=\mathbf{2 4 . 6} \mathrm{ev}$} \\
\hline
\end{tabular}

a Not included in the mean.

\section{Discussion}

The terms of $\mathrm{Zr}$ IV and $\mathrm{Zr}$ III recorded in tables 2 and 5 conform almost without exception to those required theoretically for atoms with 1 and 2 valence electrons. It is usually difficult to establish singlet terms with certainty if Zeeman patterns and intersystem combinations are missing. This is particularly true of the ${ }^{1} \mathrm{~S}_{0}$ terms of $\mathrm{Zr}$ III for which, at most, only a few combinations can occur in the spectrum. It is always possible to find, in a complex spectrum, pairs of lines that exhibit wave-number separations in approximate agreement with the separations of established levels. If any such pairs are physically real, they should satisfy further requirements relative to the intensities of the lines and the way in which they fit into series. The ${ }^{1} \mathrm{~S}_{0}$ terms in table 5 have been selected on the basis of such tests in the absence of the more convincing testimony of Zeeman observations.

A singlet term of $\mathrm{Zr}$ iII that should be accepted with reserve is the one in table 5 designated as $4 d 4 f$ ${ }^{1} \mathrm{H}_{5}^{\circ}$. It combines with the same levels as does $4 d 4 f^{1} \mathrm{G}_{4}^{\circ}$ from which it is separated by only 180 $\mathrm{cm}^{-1}$. Thus, it would violate the selection rule for inner quantum numbers if the assigned designation is correct. However, in the theoretical term-structure of the $4 d 4 f$ electron-configuration there is no room for an additional term with $J=4$. Careful combing of the spectrum for another term with $J=5$ has proved fruitless. We are left, therefore, with a choice of one of three possibilities: (1) the term labeled ${ }^{1} \mathrm{H}_{5}^{\circ}$ is correctly designated and violates the $\Delta J$ rule; $(2)$ it should be accepted as an unexplained level ${ }^{1} \mathrm{X}_{4}^{\circ}$; (3) the line at $839.55 \mathrm{~A}$ designated as $4 d$ ${ }^{3} \mathrm{~F}_{3}-4 f^{1} \mathrm{H}_{5}^{\circ}$ should be regarded as fortuitously and not physically represented by this designation. If interpretation (3) is correct this line, with appearance similar to that of nearby lines of the $4 d-4 f$ group, would be left unexplained. Choice (2) does not seem warranted. In favor of the first choice is the fact that violations of the $\Delta J$ rule are not unkown in other spectra as, for example, Paschen [6] pointed out in the case of the $\mathrm{Hg}$ II line at $2814.9 \mathrm{~A}$.

Of the isoelectronic spectra $\mathrm{Sr}$ I, Y II, Zr III, Nb IV, and $\mathrm{Mo} \mathrm{v}$, the first is the only one in which series of three or more members have been found. In the other spectra of this sequence not more than two members of a series are known. The series limits that have been reported for the spectra following Sr I have been determined either with the Rydberg formula or with the irregular-doublet law. The 
values of the limits thus derived with the one method are likely to be too high, and too low with the other. Although the limits of $\mathrm{Zr}$ In given in table 8 are undoubtedly too large, the amount by which they should be reduced can, at present, be only a matter of conjecture. Therefore, it is deemed preferable to offer these values only as provisional ones subject to revision when future data become available.

In the isoelectronic sequence of spectra from $\mathrm{Rb}$ i to Mo vi the only one with series of more than two members, following $\mathrm{Rb} \mathrm{I}$ and $\mathrm{Sr} \mathrm{II}$, is $\mathrm{Zr}$ IV. As stated above the ${ }^{2} \mathrm{~S},{ }^{2} \mathrm{~F}^{\circ}$, and ${ }^{2} \mathrm{G}$ series give concordant results in placing the ground state $4 p{ }^{2} \mathrm{D}_{11 / 2}$ of $\mathrm{Zr}$ IV $276970 \mathrm{~cm}^{-1}$ from the ground state ${ }^{1} \mathrm{~S}_{0}$ of $\mathrm{Zr} \mathrm{v}$, whereas the ${ }^{2} \mathrm{D}$ series gives a value about $18000 \mathrm{~cm}^{-1}$ greater. The series of $\mathrm{Y}$ III, Nb v, and Mo vi each consist of only two members. Although these series terms can be accurately represented with a simple Rydberg formula yet the limits calculated by this method are greater than those derived from application of the irregular-doublet rule to similar series of $\mathrm{Rb}$ I, $\mathrm{Sr}$ II, and $\mathrm{Zr}$ IV. It seems reasonable, therefore, to adopt for the limits provisional values that are means between the extreme values given by the different methods. When this is done and the results are compared with limits derived from the ${ }^{2} \mathrm{D}$ series, as in table 9 , the differences between the convergences of the series are clearly revealed. These findings confirm Russell's statement that "Series involving changes in a $d$ electron are usually very regular, except for the lowest term, when this involves the binding of the electron as part of an incomplete shell. In this case the energy of binding is considerably increased, and the application of a single Rydberg formula puts the limit a great deal too high."

The results presented in this paper could not have been achieved without the observational material supplied by Professors A. G. Shenstone, J. C. Boyce,
TABLE 9. Absolute term values of ${ }^{2} \mathrm{D}_{11 / 2}$

\begin{tabular}{|c|c|c|c|}
\hline Spectrum & $\begin{array}{c}\text { From }{ }^{2} \mathrm{~S} \text { or }{ }^{2} \mathrm{~F}^{\circ} \\
\text { series }\end{array}$ & $\begin{array}{l}\text { From }{ }^{2} \mathrm{D} \\
\text { series }\end{array}$ & Differences \\
\hline $\mathrm{Rb} \mathrm{I}$ & 14336 & $\begin{array}{l}14310^{\text {a }} \\
19780^{\text {b }}\end{array}$ & $\begin{array}{r}-26 \\
+5440\end{array}$ \\
\hline Sr II & 74408 & $\begin{array}{l}74428^{\mathrm{a}} \\
76970^{\mathrm{b}}\end{array}$ & $\begin{array}{r}+20 \\
+2560\end{array}$ \\
\hline$Y$ III & 168400 & 175080 & +6680 \\
\hline $\mathrm{Zr} \mathrm{IV}$ & 276970 & 294850 & +17880 \\
\hline $\mathrm{Nb} v$ & 407700 & 433730 & +26030 \\
\hline Mo vI & 549000 & 589630 & +40630 \\
\hline
\end{tabular}

a Calculated with high series members (Rydberg formula). b Calculated with first two series members (Rydberg formula).

and G. R. Harrison, as described above. To them I express my appreciation for their friendly cooperation, and also to C. H. Corliss, W. R. Bozman, and Miss Marion M. Harvey, of the Spectroscopy Section of the National Bureau of Standards, who assisted me with various phases of the observational and computational work of this investigation.

\section{References}

[1] C. C. Kiess and R. J. Lang, BS J. Research 5, 305 (1930) RP202.

[2] C. C. Kiess, J. Opt. Soc. Am. 44, 823 (1954); Astron. J. 58, 219 (1953).

[3] C. C. Kiess, J. Opt. Soc. Am. 43, 1024 (1953).

[4] R. W. Wood, Nature 140, 723 (1937).

[5] A. G. Shenstone, J. C. Boyce, and H. N. Russell, Rydberg interpolation table (Princeton University, 1934).

[6] F. Paschen, Akad. Wiss., Berlin, Sitz.-ber., Parts 31-33, p. 536 (1928).

[7] H. N. Russell, Astrophys. J. 66, 233 (1927).

Washington, November 8, 1955. 